\title{
Transthoracic echocardiography and mortality in sepsis: are we there yet?
}

\author{
Karan Singh* ${ }^{*}$ and Paul Mayo
}

(ㄷ) 2018 Springer-Verlag GmbH Germany, part of Springer Nature and ESICM

Critical care ultrasonography (CCUS) may be considered the fifth pillar of the physical examination [1]. A major subcomponent of CCUS is echocardiography which is in widespread use by frontline intensivists. Intensive care medicine (ICM) has been at the forefront of defining the use of CCUS [2-4].

While use of critical care echocardiography (CCE) is intuitively attractive, there are no data from randomized controlled trials (RCTs) that show improvement of mortality with use of CCE in the intensive care unit (ICU) and, in particular, for mortality of septic shock [5]. The challenge of defining meaningful outcome variables in the ICU, the difficulty of recruiting ICU teams with clinical equipoise, and the problems of developing echocardiography-driven standardized treatment protocols all combine to make it difficult to design an RCT examining the effect of CCE on mortality [5].

A potential answer to this conundrum is the use of "big data". Big data has been defined as "high-volume, high-velocity and/or high-variety information assets that demand cost-effective innovative forms of information processing that enable enhanced insight, decision making, and process automation" [6]. With widespread implementation of the electronic medical record as a source of "big data", the volume of data is enormous and exponentially growing which will help in building associations and testing hypothesis [7].

In a recent issue of this journal, Feng et al. use the "big data" approach to examine whether the utilization of echocardiography was associated with improvement in all-cause mortality of septic shock [8]. The authors utilized the MIMIC database, a large, open-access database comprising de-identified health-related data of 38,605

${ }^{*}$ Correspondence: k.singh.kahlon@gmail.com

Division of Pulmonary-Critical Care, Northwell Health, 410 Lakeville Road, Suite 107, New Hyde Park, NY 11042, USA
ICU patients of a major academic center, the Beth Israel Deaconess Medical Center in Boston (MA, USA) who were admitted between 2001 and 2012. A total of 6361 patients with septic shock met inclusion criteria of whom 51.3\% patients received a transthoracic echocardiogram (TTE) during or $24 \mathrm{~h}$ preceding their ICU admission. The decision to obtain the TTE was made by the ICU team. The study was performed by a sonography technician and interpreted by a cardiologist not part of the rounding ICU team. Using sophisticated multivariate statistical analysis, the authors observed that patients who received a TTE had lower 28-day mortality than those who did not receive one.

Sepsis may result in depression of left ventricular (LV) systolic function, hyperdynamic LV function, LV outflow obstruction (LVOT), and/or diastolic dysfunction [9-11]. It is logical to presume that identification of these forms of cardiac dysfunction with echocardiography might lead to improvement in outcomes. Using Bayesian clinical analysis, the study by Feng et al. is congruent with the high pre-test probability that TTE should improve patient outcomes. We applaud the authors for their use of a large dataset, their meticulous statistical methodology, and for their attempt to answer a clinically relevant question. While the study reinforces our belief that echocardiography is useful in the ICU, we recognize that there are methodological limitations to the study.

The study identifies an association of a TTE examination performed during a complex ICU stay with improvement of mortality. Association does not prove causality. There are alternative explanations for the reported associations between a TTE examination and mortality. The use of TTE by any particular ICU team may be a marker for management strategies that are independent of the results of the TTE (e.g. antibiotic use, ventilator management, palliative care). The dynamic physiology of the critically ill septic patient suggests that the results of a

\section{Springer}


one-time TTE cannot be representative of the complex course of these patients, so the results of the study fail the "common sense" test. It is difficult to imagine that an isolated TTE examination would have major influence on patient mortality in the ICU. The TTE exam will occasionally identify imminently life-threatening processes where immediate intervention prevents death, such as pericardial tamponade, severe LVOT obstruction, or thrombus in transit. These are few and far between. In our experience, TTE is useful to make diagnosis of processes that are not imminently life-threatening and/or to identify dysphysiology that guides changes in fluids, vasopressors, and ventilator management with a limited horizon of effect.

The authors define a pattern of consultative echocardiography where the test is ordered by the team with some delay in its performance, with delay in image interpretation, and further delay in the communication of the results to the ICU team. This is the antithesis of the way CCE should be performed where the examination is performed and interpreted by the clinician followed by immediate application of the results to bedside management. The examination may be limited in scope and repeated as often as necessary. We regret that the present study did not examine the use of TTE, as it is presently applied in an ICU staffed by CCE-capable intensivists.

The authors propose that the mortality benefit of a single TTE exam occurred in patients who were more critically ill than those who did not receive a TTE. In review, the differences between the TTE/non-TTE group for the simplified acute physiology score (SAPS) and sequential organ failure assessment (SOFA) score are rather small numerically; so, although statistically significant, they may not be clinically relevant. In the matched cohort, only the SOFA score showed a statistically significant difference. The fact that the TTE group received more fluids, dobutamine, and a higher maximal dose of norepinephrine is a not a reliable indicator that they were sicker than the non-TTE group.

In conclusion, Feng et al. report an interesting association between TTE examination and mortality in septic shock. We congratulate the authors on the use of "big data" to support use of echocardiography in the management of the critically ill. The limitations of this study underline the difficulty of studying whether echocardiography influences patient mortality. We encourage the authors in using their powerful "big data" techniques to further study the matter with focus on the current standard pattern of CCE use and to enlarge their scope of investigation to include other patient-centered outcomes such as 90-day mortality and final functional status.

Compliance with ethical standards

Conflicts of interest

None declared for both authors.

Received: 29 May 2018 Accepted: 1 June 2018

Published online: 25 June 2018

References

1. Narula J, Chandrashekhar Y, Braunwald E (2018) Time to add a fifth pillar to bedside physical examination: inspection, palpation, percussion, auscultation, and insonation. JAMA Cardiol 3:346-350. https://doi. org/10.1001/jamacardio.2018.0001

2. Expert Round Table on Ultrasound in ICU (2011) International expert statement on training standards for critical care ultrasonography. Intensive Care Med 37:1077-1083. https://doi.org/10.1007/s00134-011-2246-9

3. Cholley BP, Vieillard-Baron A, Mebazaa A (2006) Echocardiography in the ICU: time for widespread use! Intensive Care Med 32:9-10. https://doi. org/10.1007/s00134-005-2833-8

4. Mayo P, Arntfield R, Balik M et al (2017) The ICM research agenda on critical care ultrasonography. Intensive Care Med 43:1257-1269. https://doi. org/10.1007/s00134-017-4734-z

5. Mayo PH, Maury E (2014) Echography is mandatory for the initial management of critically ill patients: we are not sure. Intensive Care Med 40:1760-1762. https://doi.org/10.1007/s00134-014-3460-z

6. Zhang L, Wang H, Li Q et al (2018) Big data and medical research in China. BMJ 360:j5910

7. Austin C, Kusumoto F (2016) The application of Big Data in medicine: current implications and future directions. J Interv Card Electrophysiol 47:51-59. https://doi.org/10.1007/s10840-016-0104-y

8. Feng M, McSparron J, Trung Kienn D et al (2018) Transthoracic echocardiography and mortality in sepsis: analysis of the MIMIC-III database. Intensive Care Med. https://doi.org/10.1007/s00134-018-5208-7

9. Aneman A, Vieillard-Baron A (2016) Cardiac dysfunction in sepsis. Intensive Care Med 42:2073-2076. https://doi.org/10.1007/s00134-016-4503-4

10. Chauvet J-L, El-Dash S, Delastre O et al (2015) Early dynamic left intraventricular obstruction is associated with hypovolemia and high mortality in septic shock patients. Crit Care 19:262. https://doi.org/10.1186/s1305 4-015-0980-z

11. Sanfilippo F, Corredor C, Fletcher N et al (2015) Diastolic dysfunction and mortality in septic patients: a systematic review and meta-analysis. Intensive Care Med 41:1004-1013. https://doi.org/10.1007/s00134-015-3748-7 